\title{
TeV Scale Cross-Sections and the Pomeranchuck Singularity
}

\author{
Girish C. Joshi*
}

Research Centre of High Energy Particle Physics, University Of Melbourne, Parkville, Victoria 3010, Australia

\begin{abstract}
We have investigated the detailed structure of $l$-plane singularities of scattering amplitude saturating the Froissart bound. A self-consistent analysis of these singularities provides us secondary terms in the Froissart bound. These secondary terms lead to ghosts in the $l$-plane, which can only be removed by introducing an odderon singularity. Phenomenological implications of this analysis are also discussed.
\end{abstract}

Keywords: TeV scale, pomeron, odderon.

\section{INTRODUCTION}

TOTEM and LHCf experiments at LHC $[1,2]$ have revived new interest in the high energy behaviour of scattering cross-sections. Measurement of high-energy crosssections at energies $\sqrt{s}=14 \mathrm{TeV}$ will provide a deep insight into the dynamics of hadronic interactions and some of the most important principles of physics.

At present our theoretical understanding of physics at these energies is rather incomplete. There are a number of theories like soft QCD, eikonal and most important, the Regge theory. This theory has a remarkable history in explaining high-energy behaviour in terms of few parameters. Furthermore, with dual models like Veneziano representation, these theories provide a unified description of high-energy behaviour and low energy resonances.

In this paper we will investigate the high-energy behaviour of the Pomeranchuck singularity based on the most general principles of physics:

- Unitarity and

- Analyticity.

Our starting points will be the Froissart bound [3] and the one-dimensional dispersion relations. We will first calculate the 1-plane singularities using the Froissart bound. Then from these singularities we will derive the high-energy behaviour of the scattering amplitude. We will show that there is an interesting relationship between the detailed structure of the 1-plane singularities and the detailed structure of the highenergy behaviour. In the language of the 60 's, we are going to "bootstrap" the Pomeron.

For simplicity we start with spin zero kinematics where the t-channel Froissart-Gribov [4] representation for the partial wave amplitude is given by

$a_{l}(t)=\frac{2}{t-4 m^{2}} \int_{4 m^{2}}^{\infty} Q_{l}\left(1+\frac{2 s}{t-4 m^{2}}\right) A_{s}(t, s) d s$

*Address correspondence to this author at the Research Centre of High Energy Particle Physics, University of Melbourne, Parkville Victoria 3010, Australia; Tel: + 6183445122; Fax: +61 39347 4783;

E-mail: girish@unimelb.edu.au
This representation via Carlson's theorem [5] provides a unique interpolation to the complex angular momentum plane. In the 60's several authors [3] including this author [68] investigated the analytic properties of the partial wave amplitude $a_{l}(t)$ in the complex angular momentum plane and showed that $a_{l}(t)$ was a meromorphic function with moving poles at $l=\alpha(t)$.

In this paper we will assume Froissart bound for $A_{s}(t, s)$

$A_{s}(t, s) \leq \beta(t) \log ^{2}\left(\frac{s}{s_{0}}\right)$ for $s \geq N$

where $N$ is a large number. We can now write

$a_{l}(t)=A(l, t)+B(l, t)$

where

$$
\begin{aligned}
& A(l, t)=\frac{2}{t-4 m^{2}} \times \\
& \int_{N}^{\infty} Q_{l}\left(1+\frac{2 s}{t-4 m^{2}}\right) \beta(t) s^{\alpha(t)} \log ^{2}\left(\frac{s}{s_{0}}\right) d s
\end{aligned}
$$

and

$$
\begin{aligned}
& B(l, t)=\frac{2}{t-4 m^{2}} \times \\
& \int_{4 m^{2}}^{N} Q_{l}\left(1+\frac{2 s}{t-4 m^{2}}\right) A_{s}(t, s) d s
\end{aligned}
$$

Here $\alpha(t)$ is the Pomeranchuck trajectory with $\alpha(0)=1$ and $\mathcal{R} e \alpha(t) \leq 1$. Detailed structure of $\alpha(t)$ will be discussed later on.

In eq. (4) expanding $Q_{l}(z)$ for large $z$ we obtain

$$
\begin{gathered}
A(l, t)=2^{-1-2 l} \sqrt{\pi}\left(t-4 m^{2}\right)^{l} \frac{\Gamma(l+1)}{\Gamma(l+3 / 2)} \beta(t) s_{0}^{-2 \alpha(t)} \times \\
N^{\alpha(t)-l}\left\{\frac{1}{l-\alpha(t)} \log ^{2}\left(\frac{N}{s_{0}}\right)\right. \\
-\frac{2}{[l-\alpha(t)]^{2}} \log \left(\frac{N}{s_{0}}\right) \\
\left.+\frac{2}{[l-\alpha(t)]^{3}}\right\}
\end{gathered}
$$


The above integration is performed in the domain $l>\alpha(t)$. The resulting representation eq. (6) now provides an analytic continuation of $A(l, t)$ in the entire $l$-plane with simple, double and triple moving poles at $l=\alpha(t) . A(l, t)$ also has the usual fixed poles at $l=-1,-2, \ldots$. For the other part $B(l, t)$ we expand $A_{s}(t, s)$ in a Taylor series

$A_{s}(t, s)=\sum_{n} c_{n}(t)\left(\frac{s}{s_{0}}\right)^{n} \frac{t-4 m^{2}}{2}$

and use representation of $Q_{l}(z)$ at $z \sim 1$ to obtain

$$
\begin{aligned}
& B(l, t)=\sum_{n} c_{n}(t)\left\{\frac{\left(N / s_{0}\right)^{n+1}}{n+1} \times\right. \\
& {\left[\log \left(\frac{N}{s_{0}}\right)-2-\gamma-\psi(l+1)\right]} \\
& -\frac{\left(4 m^{2} / s_{0}\right)^{n+1}}{n+1} \times \\
& \left.\left[\log \left(\frac{4 m^{2}}{s_{0}}\right)-1+\log \left(\frac{t-4 m^{2}}{s_{0}}\right)-\gamma-\psi(l+1)\right]\right\}
\end{aligned}
$$

where $\psi(z)=\Gamma^{\prime}(z) / \Gamma(z)$ and $\gamma$ is Euler's constant. This representation $B(l, t)$ is an analytic function except for fixed poles at $l=-1,-2, \ldots$.

Now using the singularities of eq. (4) in terms of a single, double and a triple pole we can calculate the asymptotic behaviour of $A(s, t)$ via the Sommerfeld-Watson transform i.e.

$$
A(s, t)=\frac{1}{2 i} \int_{c}(2 l+1) \frac{a(l, t)}{s_{m \pi l}} P_{l}(-z) d z
$$

where the contour is clock-wise and the signature factor is included in the definition of $a(l, t)$.

Taking the residue of poles in eq.(9) we get

$$
\begin{aligned}
A(s, t) & =X Y P \log ^{2}\left(\frac{N}{s_{0}}\right) \\
& -2\left[X^{\prime} Y P+X\left(Y^{\prime} P+P^{\prime} Y\right)\right] \log \left(\frac{N}{s_{0}}\right) \\
& +X^{\prime \prime} Y P+2 X^{\prime}\left(Y^{\prime} P+P^{\prime} Y\right) \\
& +X\left(Y^{\prime \prime} P+Y^{\prime} P^{\prime}+P^{\prime \prime} Y+P^{\prime} Y^{\prime}\right)
\end{aligned}
$$

evaluated at $l=\alpha(t)$, where primes denote differential with respect to $l$ and evaluated at $l=\alpha(t)$ and

$$
\begin{gathered}
X(l, t)=\frac{2^{-1-2 l}}{2 l+1} \sqrt{\pi}\left(t-4 m^{2}\right)^{l} \times \\
\frac{\Gamma(l+1)}{\Gamma(l+3 / 2)} \beta(t) s_{0}^{-2 \alpha(t)} N^{\alpha(t)-l} \\
Y(l, t)=-\frac{1+e^{-i \pi l}}{s_{m \pi l}} \\
P=P_{l}(-z)=P_{l}\left(-1-\frac{2 s}{t-4 m^{2}}\right)
\end{gathered}
$$

At this point the high energy behaviour of the scattering amplitude as given by eq.(8) has a pathology. We call this "odderon anomaly". This comes from the $X Y^{\prime \prime} P$ term in eq.(8) which in its full form can be written as

$$
\begin{aligned}
& -X P\left[-\frac{\pi^{2}}{2}\left(\frac{1+e^{-i \pi \alpha(t)}}{\sin \pi \alpha(t)}\right)-\frac{\pi^{2}}{2}\left(\frac{1-e^{-i \pi \alpha(t)}}{\sin \pi \alpha(t)}\right)\right. \\
& +2 i \pi^{2} \frac{e^{-i \pi \alpha(t)}}{\sin ^{2} \pi \alpha(t)} \cos \pi \alpha(t) \\
& \left.+2 \pi^{2} \frac{1+e^{-i \pi \alpha(t)}}{\sin ^{3} \pi \alpha(t)} \cos ^{2} \pi \alpha(t)\right] .
\end{aligned}
$$

The first term in eq. (14) is the usual Pomeron term with positive signature. And the second term is the odd-signature Pomeron (odderon). All terms in eq. (14) are well behaved at $\alpha(0)=1$ except the odderon term, which has a ghost. Conventional ghost killing mechanisms like the Chew mechanism [9] or Gell-Mann mechanism [10] do not work here. The basic idea behind ghost-killing mechanism is that when $\alpha(0)=1$ the pole residue develops a zero at this point removing the ghost. This idea cannot work here because if $X$ develops a zero at $\alpha(0)=1$ a large number of of terms also vanish because they also have the same residue. This also removes the most important term

$$
X Y P^{\prime \prime} \sim s^{\alpha(t)} \log ^{2}\left(\frac{s}{s_{0}}\right)
$$

which is our assumption regarding the asymptotic behaviour.

However, we can remove the ghost by introducing an additional term in eq. (2) i.e.

$-X P \frac{\pi^{2}}{2}\left(\frac{1-e^{-i \pi \alpha(t)}}{\sin \pi \alpha(t)}\right) \frac{\gamma(t)}{\beta(t)}$

such that

$$
\gamma(0)=\beta(0)
$$

With this new term we can recalculate the singularities and then using the Sommerfeld-Watson [3] transform we get

$$
\begin{aligned}
& A(s, t)=\left\{X Y T \log ^{2}\left(\frac{N}{s_{0}}\right)\right. \\
& -2\left[X^{\prime} Y T+X\left(Y^{\prime} T+T^{\prime} Y\right)\right] \log \left(\frac{N}{s_{0}}\right) \\
& +X^{\prime \prime} Y T+2 X^{\prime} Y^{\prime} T+2 X^{\prime} Y T^{\prime} \\
& \left.+X\left(Y^{\prime} T+2 Y^{\prime} T^{\prime}+Y T^{\prime \prime}\right)\right\}(-z)^{\alpha(t)} \\
& +\left[-2 X Y T^{\prime} \log \left(\frac{N}{s_{0}}\right)+2 X^{\prime} Y T\right. \\
& \left.+2 X Y^{\prime} T+2 X Y T^{\prime}\right](-z)^{\alpha(t)} \log (-z) \\
& +X Y T(-z)^{\alpha(t)} \log ^{2}(-z)
\end{aligned}
$$

where

$$
P_{l}\left({ }_{z}\right)=\sqrt{\pi} \frac{\Gamma(l+1 / 2)}{\Gamma(l+1)}(-z)^{l}=T(l)(-z)^{l}
$$


evaluated at $l=\alpha(t)$ and

$T^{\prime}=\left[\frac{\partial T(l)}{\partial l}\right]_{l=\alpha(t)}$

$T^{\prime \prime}=\left[\frac{\partial^{2} T(l)}{\partial l^{2}}\right]_{l=\alpha(t)}$.

Thus using the optical theorem which is also based on unitarity

$\sigma_{\mathrm{TOT}}=\frac{8 \pi}{q \sqrt{s}} \operatorname{I} m[A(s, t=0)]$

the total cross-section can be written as

$$
\begin{aligned}
\sigma_{\mathrm{T} O T} & =16 \pi\left\{M[\alpha(0)]+N[\alpha(0)] \log \left(\frac{s}{2 m^{2}}\right)\right. \\
& \left.+X Y T \log ^{2}\left(\frac{s}{2 m^{2}}\right)\right\}
\end{aligned}
$$

with

$$
\begin{aligned}
M & =X Y T \log ^{2}\left(\frac{N}{s_{0}}\right) \\
& -2\left[X^{\prime} Y T+X\left(Y^{\prime} T+Y^{\prime}\right)\right] \log \left(\frac{N}{s_{0}}\right) \\
& +X^{\prime \prime} Y T+2 X^{\prime} Y^{\prime} T+2 X^{\prime} Y T^{\prime} \\
& +X\left(Y^{\prime \prime} T+2 Y^{\prime} T^{\prime}+Y T^{\prime \prime}\right)
\end{aligned}
$$

and

$$
\begin{aligned}
N & =-2 X Y T \log \left(\frac{N}{s_{0}}\right)+2 X^{\prime} Y T \\
& +2 X Y^{\prime} T+2 X Y T^{\prime} .
\end{aligned}
$$

where we have used the trajectory $\alpha(t)=\alpha(0)+\alpha^{\prime} t=1+\alpha^{\prime} t$.

We note that in eq. (22) there is no odderon contribution, however for small values of $t$ both Pomeron and odderon will contribute. We also note that factorisation property [1117] will not hold for eq. (22).

In eq. (16), for simplicity, we have assumed same parameters for Pomeron $\left[\alpha_{p}(t)\right]$ and Odderon $\left[\alpha_{o}(t)\right]$ trajectories. However, in general these two trajectories could have different parameters. In perturbative QCD, for example, Pomeron and Odderon are connected with different diagrams, double-gluon and triple-gluon exchanges, respectively [18-21]. Consequently their parameters are different.

These generalized QCD parameters can easily be accommodated in the present scheme. All that is needed to remove Odderon anomaly is the condition that $\alpha_{o}(0)=\alpha_{p}(0)=1$ together with eq. (17).

We will now discuss nature of Pomeranchuck trajectory. For our analysis all we need to assume is that for Pomeron $\operatorname{Re} \alpha(t) \leq 1$ and $\alpha(0)=1$. There are several examples of such trajectories like $\alpha(t)=1-\alpha^{\prime} \sqrt{-t}$

and

$\alpha(t)=1-\alpha_{1} \log \left(1+\alpha_{2} t^{2}\right)$.

It should be noted that this parameterization is valid only near $t \sim 0$. The $\mathrm{p}-\mathrm{p}$ total cross-section will also get a contribution from secondary trajectories. From the point of view of duality there are three Veneziano amplitudes $V(s, t), V(s, u)$ and $V(t, u)$. As s-channel is exotic only $V(t, u)$ will contribute. Here there are two types of mesons normal ( $\mathrm{Q} \overline{\mathrm{Q}})$ trajectories like $\rho-A_{2}$ and the baryonium trajectories (QQ Q $\bar{Q}$ ) [22-28]. As so far no baryonium are found one expects baryonium trajectories will have a smaller slope compared to $(\mathrm{Q} \overline{\mathrm{Q}})$ trajectories. Thus such trajectories will only contribute for large $t$.

A detailed phenomenological analysis of $p-p(\bar{p}-p)$ and $\pi^{ \pm}-p$ have been carried out by several authors. Donnache [29] has used a form

$\sigma_{\mathrm{TOT}}=X s^{-\varepsilon}+Y s^{-\eta}$

where the first term is the Pomeron contribution and the second term is conventional $\rho-A_{2}$ trajectories.

Block and Halzen [29] have used a form in terms of lab energy $v$

$$
\sigma^{ \pm}=c_{0}+c_{1} \log \left(\frac{v}{m}\right)+\beta_{p^{\prime}}\left(\frac{v}{m}\right)^{\mu-1} \pm \delta\left(\frac{v}{m}\right)^{\alpha-1} .
$$

These authors make a consistent fit these cross-sections. Both these forms can be obtained from our eq. (26).

\section{ACKNOWLEDGEMENT}

This work was supported by the Australian Research Council. I would like to thank Bruce McKellar, Geoff Taylor, Ray Volkas, Robert Foot and Archil Kobakhidze for interesting discussions and encouragement.

\section{CONFLICT OF INTEREST}

None declared.

\section{REFERENCES}

[1] Antchev G, Aspell P, Atanassov I, et al. Proton-proton elastic scattering at the LHC energy of $\sqrt{s}=7$ TeV.EPL 2011; 95: 41001.

[2] Antchev G. First measurement of the total proton-proton cross section at the LHC energy of $\sqrt{s}=7$ TeV.EPL 2011; 96: 21002.

[3] Froissart M. Asympotic behavour and subtractions in the Mandelstam Representation. Phy Rev 1961; 123: 1053-7.

[4] Squres EJ, Ed. Complex angular momentum and particle physics. New York: W.A. Benjamin Inc. 1963.

[5] Titchmarsh EC, Ed. Theory of functions. New York: Oxford University Press 1939

[6] Joshi GC, Banerjee H. Analytic continuation of partial wave amplitude in the complex angular momentum plane. Phys Rev 1965; 137: B1567-86.

[7] Joshi GC. Regge poles in Pi-N scattering. Phys Rev 1966; 141: 1471-8.

[8] Joshi GC. Analytic completion and Pi-N scattering. Nuovo Cimento 1965; XLA: 630-3.

[9] Chew GF. Decreasing I=0, J=0 Pi-Pi Phase shift and Regge ghosts. Phys Rev Letters 1966; 16: 60-3.

[10] Gell-Mann M. Proceedings of the international conference on high energy nuclear physics, Geneva edited by J Prentki, CERN 1962; p.539.

[11] Joshi GC. Factorization, logarithamically rising cross-section Nuovo Cimento 1975; 10: 821-5. 
[12] Joshi GC, Lo SY. Factorization in two-body reactions. Nucl Phys 1975; B93: 405-14.

[13] Joshi GC, Kellett BH, Lo SY. Factorization in inclusinve reactions. Nuovo Cimento 1977; 41 A: 331-50.

[14] Joshi GC, Lo SY, Kellett BH. Factorization in the central region of inclusive reactions. Nuovo Cimento 1979; 47A: 281-6.

[15] Joshi GC, Kellett BH, Lo SY. Factorization in inclusive reactions II. Nuovo Cimento 1977; 41 A: 351-8.

[16] Joshi GC, Martin AW. 1 -plane and Khuri plane singularities in Veneziano model. Phys Rev 1969;188: 2354-61.

[17] Joshi GC, Pagnamenta A. Veneziano amplitude for Pi-N Scattering. Phys Rev 1970; D 1: 3117-23.

[18] Brodsky SJ, Fadin VS, Kim VT, Liptov LN, Pivovarov GB. Highenergy QCD asymptotics of photon-photon collisions. JETP Lett 1999; 70: 155-60.

[19] Brodsky SJ, Hautmann JF, Soper DE. BFKL scattering at LEP-2 and a next e+ e- collider. Phys Rev 1997; D56: 6957-79.

[20] Merino C, Brodsky SJ, Rathsman J. Jet asymmetry and the detection of odderon exchange in DIS. J Nucl Phys Proc 2000; Suppl. 86: 183-8.
[21] Merino C, Brodsky SJ, Rathsman J. Odderon-pomeron interference. Phys Lett 1999; B461: 114-22.

[22] Joshi GC, Pagnamenta A. Dual model for N - N and N - -N scattering. Part Fields 1970; 1: 220-7.

[23] Joshi GC, Anderson RCG. Coefficients for Baryonium States. J Math Phys 1979; 20: 1015-29.

[24] Joshi GC, Warner R. Phenomenology of quarkonium. Hadronic J 1979; 20: 198-237.

[25] Joshi GC, Anderson R. Mass degeneracy in Baryonium spectrum. Phys Rev 1979; 20: 736-42.

[26] Joshi GC, Warner R. Four-body potential in multiquark states. Phys Rev 1980; 22: 1012-7.

[27] Ellis RG, Anderson R, Joshi GC, McKellar BHJ. Electromagnetic widths for $\mathrm{L}=1$, JPC $=1$-- T-Baryonia. Phys Rev 1980; D 22: 28327.

[28] Ellis RG, McKellar BHJ, Joshi GC. T and M-Baryonia from e+eexperiments. Nuovo Cimento Lett 1981; 30: 455-60.

[29] Block MM, Halzen F. New evidence for the saturation of the Froissart bound. Phys Rev 2005; D 72: 036006-10.

(C) Girish C. Joshi; Licensee Bentham Open.

This is an open access article licensed under the terms of the Creative Commons Attribution Non-Commercial License (http://creativecommons.org/licenses/by$\mathrm{nc} / 3.0 /$ ) which permits unrestricted, non-commercial use, distribution and reproduction in any medium, provided the work is properly cited. 